\title{
Relaxation dynamics of water-immersed granular avalanches
}

\author{
DELPHINE DOPPLER ${ }^{1}$, PHILIPPE GONDRET ${ }^{1}$, \\ THOMAS LOISELEUX ${ }^{1,2}$, SAM MEYER ${ }^{1}$ \\ AND MARC RABAUD ${ }^{1}$ \\ ${ }^{1}$ Lab FAST, UMR 7608, CNRS, Univ Paris-Sud, Université Pierre et Marie Curie-Paris 6, \\ Bât. 502, F-91405 Orsay, France \\ ${ }^{2}$ Unité de Mécanique, Ecole Nationale Supérieure de Techniques Avancées \\ (ENSTA-ParisTech), 32 Bd Victor, F-75015 Paris, France
}

(Received 26 April 2006 and in revised form 23 October 2006)

We study water-immersed granular avalanches in a long rectangular cell of small thickness. By video means, both the angle of the granular pile and the velocity profiles of the grains across the depth are recorded as a function of time. These measurements give access to the instantaneous granular flux. By inclining the pile at initial angles larger than the maximum angle of stability, avalanches are triggered and last for a long time, up to several hours for small grains, during which both the slope angle and the granular flux relax slowly. We show that the relaxation is quasi-steady so that there is no inertia: the relaxation at a given time is controlled only by the slope angle at that time. This allows us to adapt a frictional model developed recently for dry or water-immersed grains flowing in stationary conditions. This model succeeds well in reproducing our unsteady avalanche flows, namely the flowing layer thickness, the granular flux and the temporal relaxation of the slope. When a water counter-flow is applied along the pile, the granular avalanches are slowed down and behave as if granular friction were increased by an amount proportional to the water flow. All these findings are also reproduced well with the same friction model by taking into account the additional fluid force.

\section{Introduction}

Although the flow equations for simple fluids have been known for more than a century, this is not true for granular matter. This is why many experimental studies similar to those in the past for water flows have been conducted in recent years for granular flow, together with numerical studies, in order to obtain insight into and validate the different proposed theoretical approaches. Granular flow may sometimes resemble that of a liquid but its detailed behaviour is much more complex, in particular the hysteretic behaviour that leads it to switch from a static to a flowing state and vice versa.

Good knowledge of granular avalanche flows is crucial in a large number of industrial and environmental problems. In all these practical configurations, the interstitial fluid can be a gas or a liquid. The influence of the interstitial fluid is clearly important in the granular avalanche process, as evidenced by the marked differences between the propagation of aeolian and submarine dunes: the flow may be continuous at the lee side of submarine dunes, but occurs by successive avalanches 
for aeolian dunes (Hunter 1985). This is why two geologists, Allen (1970) and Carrigy (1970), made the first comparative studies between dry and water-immersed granular avalanches in a slowly rotating drum, focusing on the avalanche duration $T$ and the avalanche amplitude $\Delta \beta=\beta_{c}-\beta_{r}$ that corresponds to the difference between the critical angle $\beta_{c}$ at which an avalanche starts and the angle of repose $\beta_{r}$ at which an avalanche stops. They concluded that the amplitude and time duration of waterimmersed granular avalanches are respectively smaller and larger than for the dry case.

In recent years, much attention has been paid to the dry case, and a review of numerous different studies can be found in GDR MiDi (2004). All the experimental and numerical results collected in this paper suggested that a local rheology, with a dynamical friction coefficient $\mu$ depending on a dimensionless shear rate, correctly describes the steady avalanche flows down inclined planes. This dimensionless shear rate, named the inertial number and denoted $I$, arised from the work of da Cruz et al. (2005) on the steady plane shear configuration. The inertial number $I$ can be viewed as the ratio of the typical falling time of one grain over the distance of its diameter (due to the confining external pressure or gravity) to the characteristic time of shear (GDR MiDi 2004). Recently, the crucial role of sidewalls in steady granular surface flow on a heap has been investigated experimentally by Jop, Forterre \& Pouliquen (2005): the strong localization of the shear at the heap surface can be recovered by the same local rheology $\mu(I)$ provided that solid friction at the nearby walls is taken into account. Note that a similar local rheology has been developed in the meantime by Josserand, Lagree \& Lhuillier (2004) with a slightly different point of view: the friction coefficient $\mu$ depends not only on $I$ but also on the confining pressure, while the local solid packing fraction $\phi$ is also a function of these two quantities. These two examples of local rheology appear to be able to reproduce many experimental observations for stationary conditions. These successes do not mean that the rheology is purely local since some non-local behaviours, such as arching effects, are clearly seen (see e.g. GDR MiDi 2004) and that non-local modelling can also succeed in reproducing some of the experimental observations (Mills, Loggia \& Texier 1999; Andreotti \& Douady 2001; Rajchenbach 2003).

Some recent studies have focused on the role of the interstitial fluid in dense granular flows. Courrech du Pont et al. (2003a) have systematically studied the avalanche duration and amplitude for different bead sizes, different bead and fluid densities, and different fluid viscosities, in a slowly rotating drum. They found three different regimes depending on two dimensionless parameters: the grain/fluid density ratio $r$ and the Stokes number $S t$ that corresponds to the ratio of grain inertia to fluid dissipation. In the 'free-fall' regime at large $r$ and $S t$ values corresponding typically to solid grains immersed in a gas (e.g. the so-called dry case) the interstitial fluid can be neglected and the avalanche duration scales as the free-fall of one grain along the pile length $L$. Otherwise, the interstitial fluid controls the dynamics: at low St (small grains in viscous fluids, e.g. fine sand in water), the regime is 'viscous' as viscous forces are predominant, and the avalanche duration scaling corresponds to the pile length $L$ covered by one grain at its terminal viscous Stokes velocity; at low $r$ and large enough St (large grains in liquids, e.g. coarse sand in water), the regime is 'inertial' as inertial fluid forces dominate, and the avalanche duration scaling corresponds to the pile length $L$ covered by one grain at its terminal inertial velocity. In a fast rotating drum, Jain, Ottino \& Lueptow (2004) studied by particle tracking velocimetry (PTV) the steady flow of large steel beads in air, water or different waterglycerine mixtures and drew the main following conclusion: the velocity profile in the liquid case has a similar shape as in the dry case. By using an inclined recirculating 


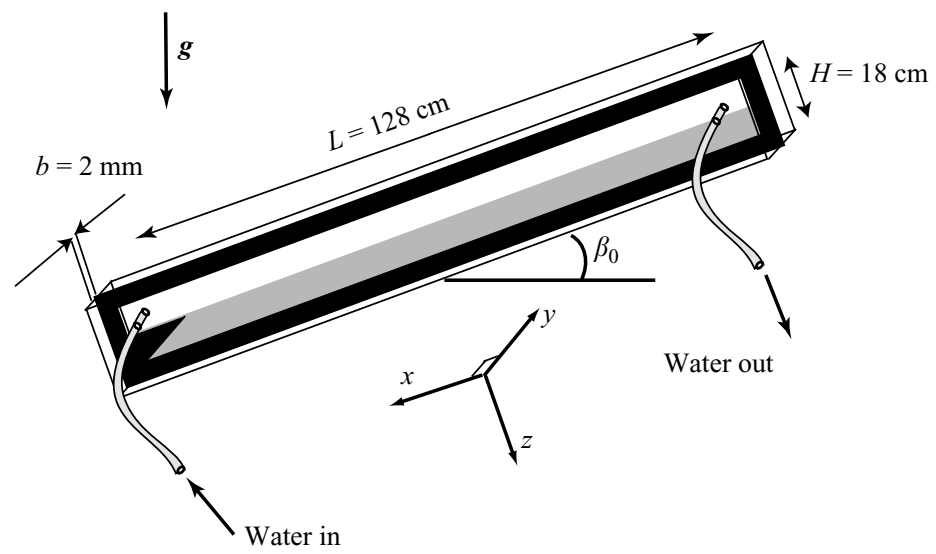

FIGURE 1. Sketch of the tiltable experimental Hele-Shaw cell.

flume, Armanini et al. (2005) tracked the steady flow of PVC pellets in water using a Voronoi imaging method. They found that the flow organizes into different sublayers with either frictional or collisional behaviour. The transition between the frictional behaviour at low shear rate and the collisional behaviour at high shear rate appears to be controlled by the Stokes number, which has been shown to be the pertinent parameter that governs the liquid-immersed collision of grains (Davis, Serayssol \& Hinch 1986; Joseph et al. 2001; Gondret, Lance \& Petit 2002). More recently Cassar, Nicolas \& Pouliquen (2005) investigated experimentally the steady flow of glass beads in water down a rough inclined plane. They successfully analysed their results within the local rheology framework $\mu(I)$ developed initially in the dry case but adapted for the liquid-immersed configuration: the falling time scale that enters the definition of the inertial number $I$ is here the viscous falling time already suggested by Courrech du Pont et al. (2003a). Finally, Loiseleux et al. (2005) studied the influence of a superficial water flow on the onset of granular avalanches: an upward water flow tends to increase the pile stability while a downward water flow decreases it.

In this paper, we report results on the unsteady relaxation dynamics of a pile of small glass beads immersed in water in a long and thin inclinable channel, in the viscous avalanche regime defined by Courrech du Pont et al. (2003a). The paper is organized as follows. The experimental set-up and measurement methods are presented in $\S 2$. The experimental results for the slope relaxation, granular flux, velocity profile and flowing layer thickness are presented and discussed in $\S 3$, together with the influence of a water counter-flow. Our experimental configuration is then modelled in $\S 4$ by using the local rheology $\mu(I)$. At the end of $\S 4$, we add a term to the model to take into account the effect of a water counter-flow. All these model predictions are compared to the experimental data and discussed.

\section{Description of the experiments}

\subsection{Set-up}

The experimental set-up is composed of two $10 \mathrm{~mm}$ thick glass plates separated by a thin rubber sheet which defines the perimeter of the channel (length $L=128 \mathrm{~cm}$ and height $H=18 \mathrm{~cm}$ ) and ensures a constant gap $b=2.0 \pm 0.1 \mathrm{~mm}$ (figure 1). This Hele-Shaw cell is half filled with spherical glass beads (density $\rho_{g}=2.5 \times 10^{3} \mathrm{~kg} \mathrm{~m}^{-3}$ ) 
manually sieved to be within the range $[125,140] \mu \mathrm{m}$, with mean diameter $d \simeq 132 \mu \mathrm{m}$. As the ratio between the width of the channel and the diameter of the beads $b / d \simeq 15$ is not large, owing to solid wall friction, the two characteristic pile angles $\beta_{c}$ and $\beta_{r}$ are increased by a few degrees compared to the values for non-confined piles (Courrech du Pont et al. 2003b). The particles are completely immersed in de-ionized water (density $\rho=10^{3} \mathrm{~kg} \mathrm{~m}^{-3}$ and viscosity $\eta=10^{-3} \mathrm{~Pa} \mathrm{~s}$ ). With such grains and fluid, one can calculate the Stokes velocity for one isolated falling grain $V_{S t}=\Delta \rho g d^{2} / 18 \eta$ where $\Delta \rho=\rho_{g}-\rho$ is the density difference between grains and liquid, and also calculate the density ratio $r=\left(5 \rho_{g} / 3 \rho\right)^{1 / 2}$ and the Stokes number $S t=\rho_{g}^{1 / 2} d^{3 / 2}(\Delta \rho g)^{1 / 2} / 18 \sqrt{2} \eta$ defined by Courrech du Pont et al. (2003a) for determining the avalanche regime: $V_{S t} \simeq 14 \mathrm{~mm} \mathrm{~s}^{-1}, r \simeq 2$ and $S t \simeq 0.4$. As $r<5$ and $S t / r<2$, the avalanche regime will be viscous. The cell is connected to a hydraulic pump which, imposing a pressure difference between the inlet and outlet of the cell, gives rise to a continuous steady laminar water flow above the granular bed. As the water flow rate through the porous granular bed is negligible (Beavers \& Joseph 1967), the mean water flow rate $Q_{f}$ above the granular bed is measured with a flow meter. In a Hele-Shaw cell, Gondret et al. (1997) showed that, with flat and rigid walls, the velocity profile of the flowing liquid is parabolic in the transverse direction ( $y$-axis), and constant in the central $(x, z)$-plane except for an exponential decrease near the top and bottom walls. Thus the water flow rate $Q_{f}$ gives the mean velocity $\overline{U_{f}} \simeq Q_{f} /\left(b h_{f}\right)$ where $h_{f}$ is the water height $\left(h_{f} \sim H / 2\right)$. The shear stress exerted by the fluid on the granular interface can finally be inferred from the relation $\tau_{x z} \simeq 3.26 \eta \overline{U_{f}} / b$ (cf. the appendix of Loiseleux et al. 2005).

The particles are backlighted with a white LED box and video cameras are fixed to the channel. The whole set-up, mounted on a motorized device, is tiltable in its $(x, z)$-plane around the horizontal $y$-axis from $-60^{\circ}$ to $+60^{\circ}$ without shocks with an angular velocity of about $10^{\circ} \mathrm{s}^{-1}$. The angle between the longitudinal axis of the experimental cell $O x$ and the horizontal is measured with a digital inclinometer with a precision of $\pm 0.1^{\circ}$. More details about the set-up can be found in Doppler (2005).

\subsection{Measurements}

We restrict our study here to granular flows in still water, or with a weak water flow acting against gravity (counter-flow). The experimental procedure is the following. We first carefully prepare a flat granular interface by alternately tilting the cell to large opposite angles, thus triggering avalanches in both directions. An experiment starts when the granular bed is tilted rapidly from slightly below $\beta_{r}$ up to a fixed angle $\beta_{0}>\beta_{c}$ within roughly $1 \mathrm{~s}$. An avalanche starts and the pile slope relaxes slowly toward the angle of repose $\beta_{r}$. During the avalanche, which can last a few hours, the interface remains flat, and the avalanche dynamics is thus characterized well by the time evolution of the slope angle $\beta(t)$ from $\beta_{0}$ to $\beta_{r}$. When a water counter-flow is imposed, it is applied just before tilting the cell. In that case, the angles $\beta_{c}$ and $\beta_{r}$ are slightly larger as described in $\S 3.2$.

The relaxation dynamics of the granular pile is studied experimentally using both global and local measurements.

In the global measurement technique, we focus on the relaxation time of the free surface of the pile. A CCD camera is placed at midlength and images are grabbed at a frequency of $1 \mathrm{~Hz}$. As the channel is closed and contains a fixed quantity of grains, the accumulation of particles at the base of the pile causes the downstream height to increase, whereas the upstream height decreases but the interface remains flat during the avalanche. By image processing we extract the granular interface and 
then calculate its instantaneous mean slope $\beta(t)$. By mass conservation, the granular flux per unit width $q_{\beta}$ at the centre of the pile can be inferred from the evolution rate of the surface slope $\mathrm{d} \beta / \mathrm{d} t$ by the relation

$$
q_{\beta}=-\frac{L^{2}}{8} \frac{\mathrm{d} \beta}{\mathrm{d} t} .
$$

This granular flux $q_{\beta}$ per unit width is related to the effective mass flow rate of the grains $Q_{m}$ by $Q_{m}=\phi \rho_{g} b q_{\beta}$, where $\phi$ is the volume solid fraction of the granular packing.

In the local measurement technique, we measure the velocity field of the particles close to the wall with a particle image velocimetry (PIV) method based on correlation techniques between two successive images which provides good measures of grain velocity fields (Lueptow, Akonur \& Shinbrot 1999). For this, another camera with high image rates (from 100 to 1000 frames s$^{-1}$ ) is placed at the midlength of the cell. Video sequences of about $10 \mathrm{~s}$ length are recorded, and then analysed with commercial PIV software. We use interrogation windows $(12 \times 12$ pixels $)$ corresponding roughly to one grain size, which gives the velocity field at the grain scale. To reduce noise, the velocity fields are averaged in the flow direction over the whole image width (typically $7 \mathrm{~mm}$ ) and averaged in time over typically 50 successive images. The compromise between temporal and spatial resolutions as well as PIV parameters gives a final velocity accuracy of about $0.1 \mathrm{~mm} \mathrm{~s}^{-1}$ and a depth resolution of $0.1 \mathrm{~mm}$ corresponding to one grain diameter. Note that we observe the velocity field of the particles only at the wall and not in the bulk. However Courrech et al. (2005) showed that the dynamical evolution of gap-averaged velocities and wall velocities is comparable. With this PIV method we extract the grain velocity profiles $v(z)$ across the depth for different initial tilting angles $\beta_{0}$ with or without a water counter-flow, and we obtain, using numerical integration, the granular flux $q_{p i v}=\int v(z) \mathrm{d} z$ at the wall. The granular flux $q_{p i v}$ is measured at the wall and is therefore related to $q_{\beta}$ by a coefficient that depends on the transverse profile of the velocity, more precisely on its exact form and slip velocity at the wall (§4.1.7). The two granular flux measurements $q_{\beta}$ and $q_{p i v}$ will be compared in the following.

\section{Experimental results}

Results obtained without an imposed water counter-flow are detailed in $\S 3.1$. The effect of a water counter-flow is then presented in $\S 3.2$.

\subsection{Quasi-steady granular flows}

\subsubsection{Slope relaxation}

The time evolution of the pile slope $\beta(t)$ is presented in figure 2 for various initial tilt angles $\beta_{0}$ ranging roughly from the mean maximum angle of stability $\beta_{c}\left(\sim 31^{\circ}\right)$ up to $39^{\circ}$ for which the system is far from equilibrium. In each case, the surface slope decreases in time but more and more slowly. The avalanches end after a few hours at an angle of repose $\beta_{r}$. As it is usually observed for avalanches, the final slope angle differs slightly from one experiment to another: the angle of repose fluctuates by typically $1^{\circ}$ around its mean value: $\beta_{r} \simeq 29 \pm 1^{\circ}$. It is worth noting that the time variation of the slope angle $\mathrm{d} \beta / \mathrm{d} t$ appears to be correlated with the slope angles $\beta$ : it is larger for larger angles. Note that for very large $\beta_{0}$ angles $\left(\beta_{0}>45^{\circ}\right)$, the interface is no longer flat, with downward propagating waves and particles in suspension. 


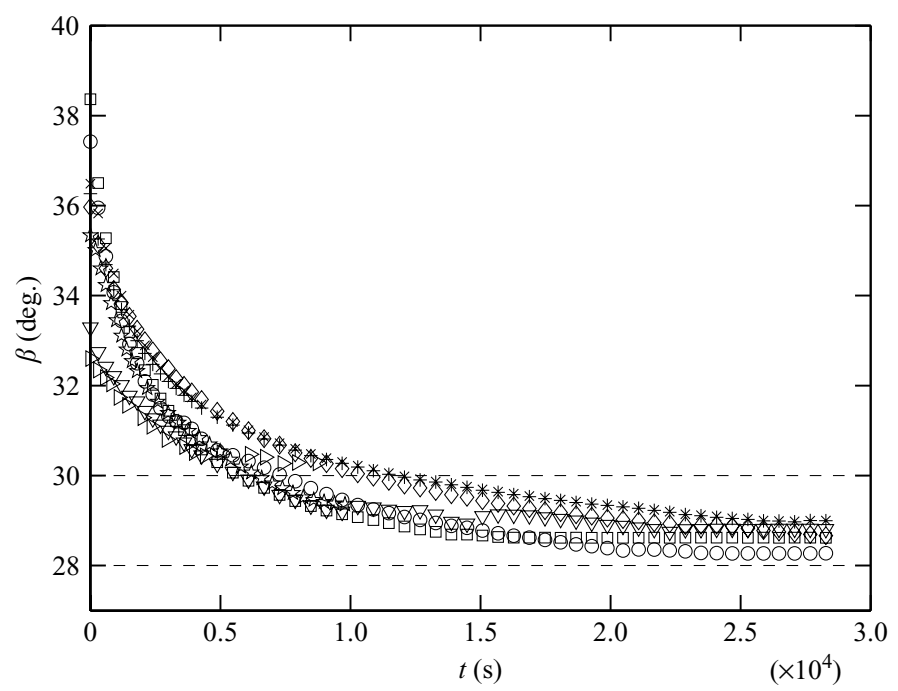

FIGURE 2. Time evolution of the pile slope $\beta$ for different initial tilt angles $\beta_{0}: \triangleright, \beta_{0}=32.6^{\circ}$; $\nabla, \beta_{0}=33.3^{\circ} ;$ 功, $\beta_{0}=35.4^{\circ} ; \diamond, \beta_{0}=36.0^{\circ} ;+, \beta_{0}=36.3^{\circ} ; \times, \beta_{0}=36.5^{\circ} ; \circ, \beta_{0}=37.4^{\circ} ; \square$, $\beta_{0}=38.4^{\circ}$. - - , limit values of $\beta_{r}=29^{\circ} \pm 1^{\circ}$.
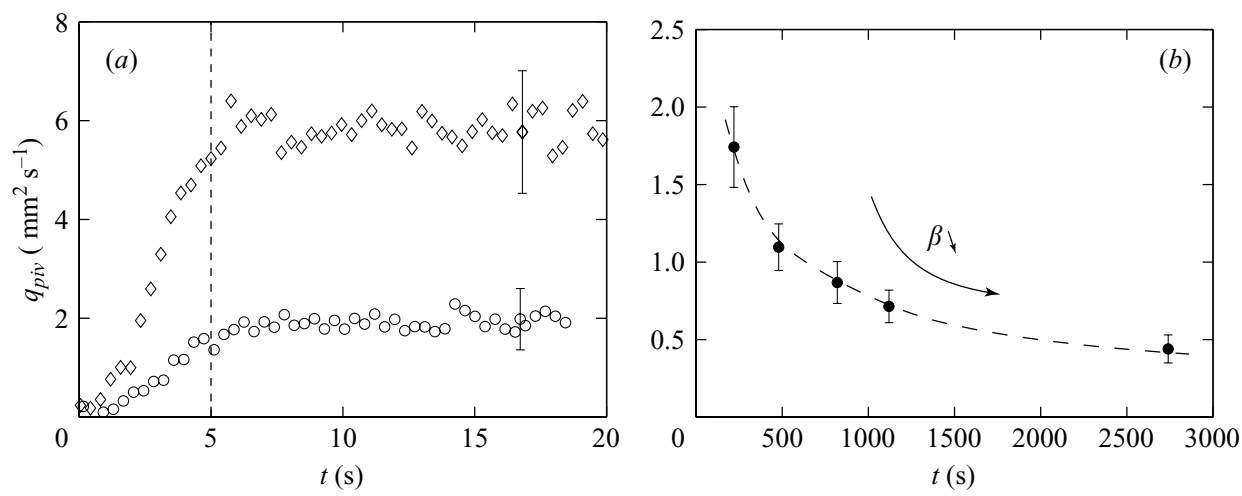

FIgURE 3. Time evolution of the granular flux $q_{\text {piv }}$ measured by PIV means at $(a)$ short and (b) long times. Experimental data are averaged over 40 images: data points correspond to mean values and error bars to typical standard deviations. $(a) \beta_{0}=31.2^{\circ}(\bigcirc), \beta_{0}=33.0^{\circ}(\diamond)$; (b) $\beta_{0}=31.6^{\circ}(\bullet)$. Dashed lines are a guide for the eyes.

\subsubsection{Granular flux}

The time evolution of the granular flux $q_{\text {piv }}$ obtained from PIV measurements is presented for short times in figure 3(a). Data show that in the first few seconds of the avalanche the granular flux increases before rapidly saturating at a plateau value. The granular flux, which can be viewed as saturated at short times when the slope angle does not change significantly, decreases slowly in time at larger time as the avalanche continues and the angle slowly relaxes (figure $3 b$ ). The time duration of the short acceleration stage is about $5 \mathrm{~s}$, and does not vary significantly with $\beta_{0}$, whereas the avalanche lasts typically few hours. Two time scales can thus clearly be distinguished: the granular flux adapts itself in about $5 \mathrm{~s}$ to any $\beta$ variation and after this transient, the granular flux appears to be correlated with the instantaneous pile slope $\beta$. We can thus 


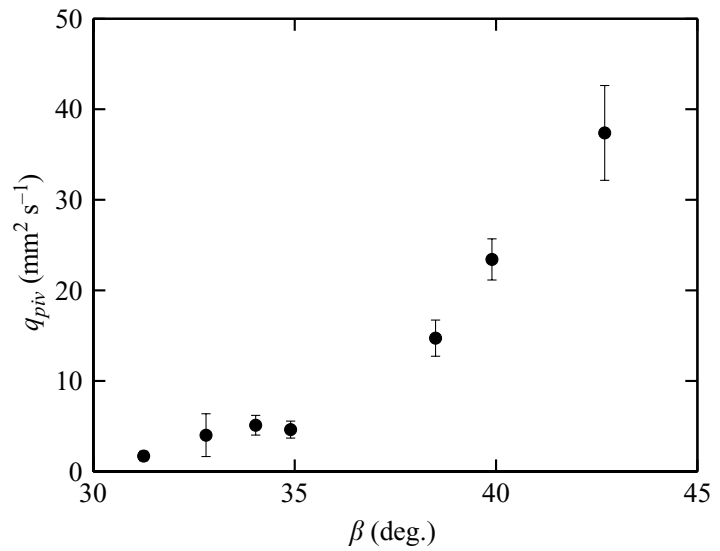

FIgURE 4. Granular flux $q_{\text {piv }}$ as a function of the pile angle $\beta$. Data points correspond to mean values and error bars to standard deviations.
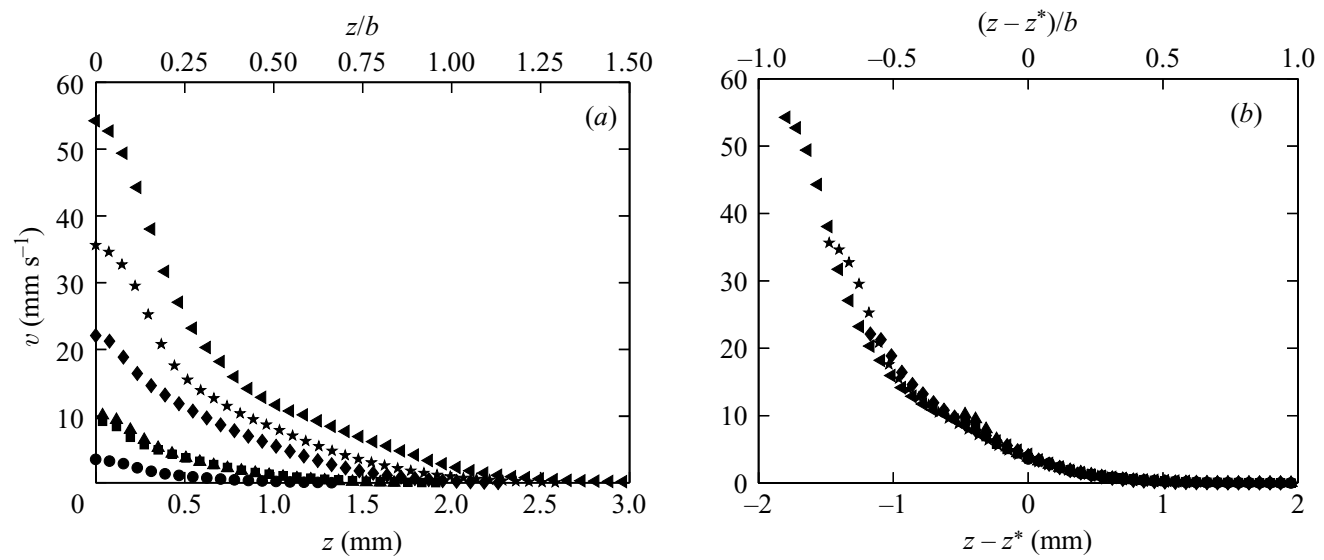

FiguRE 5. (a) Grain velocity profiles over depth $z$ for different pile angles $\beta$ : $\bullet, \beta=31.3^{\circ}$; $\mathbf{\square}, \beta=34.3^{\circ} ; \boldsymbol{\Lambda}, \beta=34.9^{\circ} ; \diamond, \beta=38.5^{\circ} ; \star, \beta=39.9^{\circ} ; \varangle, \beta=42.7^{\circ}$. (b) Same data shifted in depth by a quantity $z^{*}$ such that $v\left(z^{*}\right)=4 \mathrm{~mm} \mathrm{~s}^{-1}$.

conclude that after the quick transient the avalanche flow is quasi-steady without inertia effects. This result is not surprising as it is in the viscous regime defined by Courrech du Pont et al. (2003a). For different initial pile angles $\beta_{0}$, we measure the maximum saturated granular flux $q_{\text {piv }}$ (figure 4). As expected from the time decrease of $\beta$ (figure 2) and $q_{\text {piv }}$ (figure $3 b$ ), the measurements of figure 4 show that $q_{p i v}$ increases with $\beta$.

\subsubsection{Velocity profiles}

The velocity profiles $v(z)$ measured across the depth in the quasi-steady flow regime for various pile slopes $\beta$ are shown in figure 5(a). Whatever the pile slope, the velocity is maximum at the pile surface $(z=0)$ and decreases toward zero at increasing depth. Data show that only a thin surface layer, roughly 1 to $2 \mathrm{~mm}$ thick, flows significantly. The surface velocity increases with the pile slope, taking values up to $55 \mathrm{~mm} \mathrm{~s}^{-1}$, of the same order as the values found by Cassar et al. (2005) in the inclined rough plane configuration. These values can be larger than the Stokes velocity $V_{S t} \simeq 14 \mathrm{~mm} \mathrm{~s}^{-1}$ of a single falling isolated grain since the avalanche corresponds to a collective motion. 


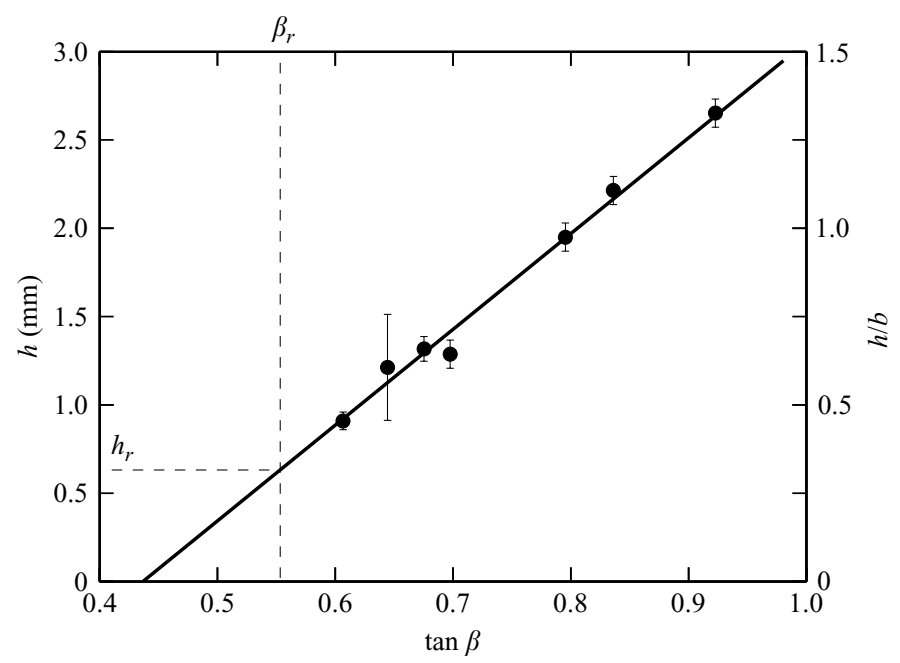

Figure 6. Flowing layer thickness $h$ as a function of the pile slope $\beta$. $\bullet$, Experimental data together with error bars corresponding to one standard deviation. The solid line corresponds to the linear fit $h(\mathrm{~mm})=5.4(\tan \beta-0.43)$.

Our profiles are similar to those already observed for steady surface flows in the dry case (Bonamy, Daviaud \& Laurent 2002; GDR MiDi 2004) or liquid-immersed case (Jain et al. 2004). By shifting each velocity profile in $z$ by a value $z^{*}$ depending on the slope angle $\beta$, all data of figure $5(a)$ collapse rather well on a single curve as shown in figure 5(b). As shown by Bonamy et al. (2002) and GDR MiDi (2004), this master curve has a roughly linear part near the surface and an exponential tail further down. During the slow quasi-steady slope relaxation, the grain velocity is slowly decreasing and the velocity profile therefore corresponds to a smaller and smaller part of the master curve. In particular, for $\beta<\beta_{c}$, the velocity profile is only exponential, as shown by Courrech et al. (2005) for dry natural avalanches.

\subsubsection{Flowing layer thickness}

From the velocity profiles, we define the thickness $h$ of the flowing layer at the depth where the velocity is larger than a cutoff velocity $\left(\sim 0.1 \mathrm{~mm} \mathrm{~s}^{-1}\right.$ corresponding to the less precise measurement). The measurements of $h$ for various experiments are reported in figure 6 as a function of the tilt angle. This figure shows that the flowing layer thickness $h$ increases linearly with $\tan \beta$. Similar results are found for larger values of the cutoff velocity. Such a result has already been observed by Taberlet et al. (2003) and Jop et al. (2005) for steady dry granular flows with continuous feeding, and has been attributed to wall effects. In our case, the measured flowing layer thickness varies roughly from 1 to $3 \mathrm{~mm}$ ( 5 to 20 grain diameters) which is of the order of the gap thickness $b=2 \mathrm{~mm}$. Note that no data are reported below the angle of repose $\beta_{r}$ as no flow occurs then. Thus a non-zero flowing layer thickness may exist at the flowing/static transition, here $h_{r} \simeq 0.7 \mathrm{~mm}$ at $\tan \beta_{r} \simeq 0.55$.

\subsection{Effect of a counter-flow}

We now report the effect of a water counter-flow imposed along the granular pile on the avalanches. The water flow rate is chosen below the threshold corresponding to the onset of bed-load motion. Loiseleux et al. (2005) have shown that this onset corresponds to a critical value $\theta_{c}$ of the Shields number $\theta=\tau_{x z} /(\Delta \rho g d)=$ $3.26 \eta \overline{U_{f}} /(\Delta \rho g b d)$, that compares the fluid forces acting on a surface grain to its 


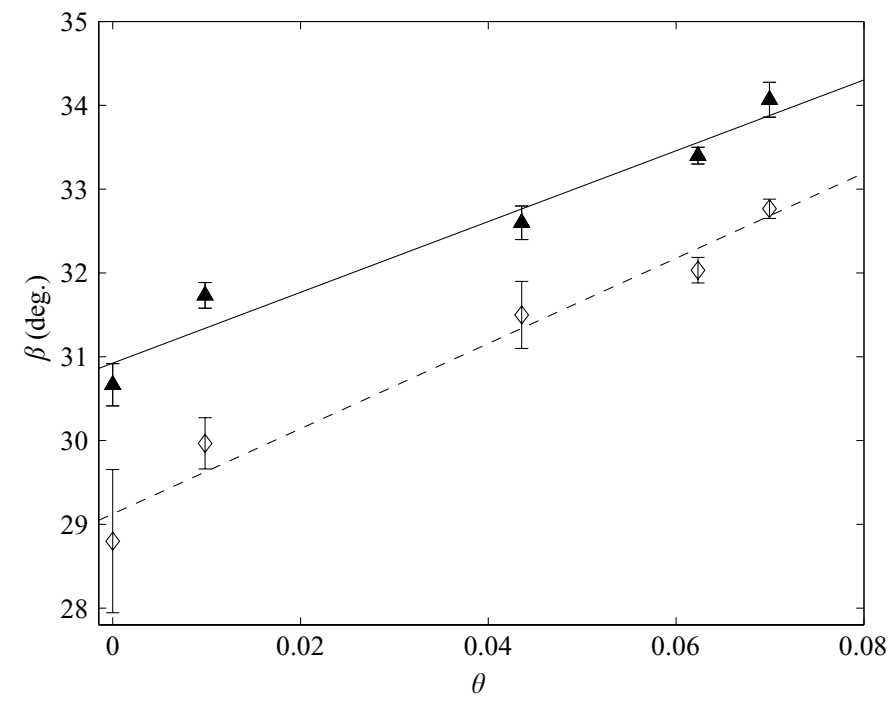

FIGURE 7. Experimental maximum angle of stability $\beta_{c}(\mathbf{\Delta})$ and angle of repose $\beta_{r}(\diamond)$ as a function of the Shields number $\theta$ for water counter-flows $(\theta>0)$, with best linear fits $\beta_{c}(\theta)=30.9+42.2 \theta(-), \beta_{r}(\theta)=29.1+50.8 \theta(--)$.

apparent weight. When both avalanche and counter-erosion processes take place simultaneously, Loiseleux et al. (2005) also mentioned that nonlinear structures grow quickly. In all the present experiments, the $\theta$ value will thus be kept below the critical value 0.1 to avoid any bed-load motion and formation of structures that would drastically modify the avalanche flow. We also restrict our study to water counterflow as it is difficult to distinguish between erosion and avalanche granular motions for water co-current flows (Loiseleux et al. 2005).

\subsubsection{Angles of stability}

The water flow modifies the force equilibrium acting on the pile and thus the two characteristic angles $\beta_{c}$ and $\beta_{r}$. For different flow rates, i.e. different values of the Shields number, we measured the corresponding maximum angle of stability $\beta_{c}$ and angle of repose $\beta_{r}$. The influence of the Shields number $\theta$ on both angles is presented in figure 7 for the grain diameter studied here $(d \simeq 132 \mu \mathrm{m})$. As observed for various other diameters (Loiseleux et al. 2005), both $\beta_{c}$ and $\beta_{r}$ increase linearly with $\theta$ by a few degrees, typically from $31^{\circ}$ to $34^{\circ}$ for $\beta_{c}$ and from $29^{\circ}$ to $33^{\circ}$ for $\beta_{r}$.

\subsubsection{Avalanche dynamics}

When a water counter-flow is imposed along the pile, the relaxation process of the pile angle is slowed down but remains similar to that without any water flow. We find that the time needed for the granular flux to reach a steady state is the same without or with a counter-flow (Doppler 2005). More strikingly, it is observed that velocity profiles measured in the quasi-steady regime, without or with a counter-flow, present similar shapes, as illustrated in figure $8(a)$. For a given slope angle $\beta$, e.g. $\beta=42.6^{\circ}$ $(\tan \beta=0.92)$ in figure $8(a)$, the water counter-flow tends to slow down the grains. However, as shown in figure $8(a)$, velocity profiles coincide for the same deviation from the maximum angle of stability $\tan \beta-\tan \beta_{r}(\theta)$, e.g. $\tan \beta-\tan \beta_{r}(\theta)=0.28$ in figure $8(a)$. Such a collapse is observed for various data sets.

The thickness $h$ of the granular flowing layer is shown in figure $8(b)$ to also increase linearly with $\tan \beta$ when a counter-flow is applied (open symbols). For a given angle 

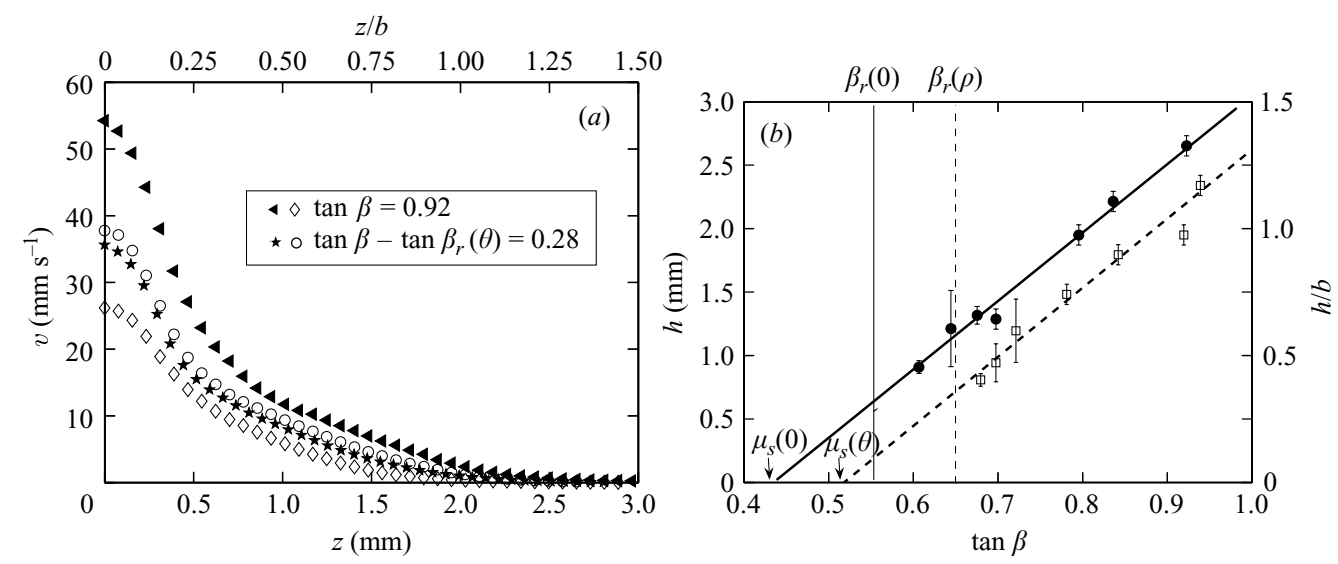

FIGURE 8. (a) Velocity profiles over depth $z$ for different values of $\beta$ with the counter-flow $\theta=0.08\left(\beta=42.6^{\circ}(\diamond), \beta=43.2^{\circ}(\circ)\right)$ or without counter-flow $\left(\beta=39.9^{\circ}(\star), \beta=42.7^{\circ}(\triangleleft)\right)$. (b) Flowing layer thickness $h$ as a function of the pile angle $\beta$ with the counter-flow $\theta=0.08(\square)$ or without counter-flow $(\bullet)$. Best linear fits give $h(\theta)=5.4(\tan \beta-0.51)$ with the counter-flow $\theta=0.08(--)$, and $h(0)=5.4(\tan \beta-0.43)$ without counter-flow $(-)$.

$\beta, h$ is reduced when a water counter-flow is applied however. Again, this is due to the resisting effect of the counter-flow that slows down the avalanche. As the slope of the two linear fits of figure $8(b)$ is the same, the $h$ reduction is due to a larger intercept with the horizontal axis corresponding to a larger static friction coefficient (see $\S 4$ ); here $\mu_{s}(\theta)=0.51$ for $\theta=0.08$ when compared to the no-water-flow case, $\mu_{s}(0)=0.43$ for $\theta=0$. Note that the difference $\mu_{s}(\theta)-\mu_{s}(0) \simeq 0.08$ for the friction coefficient appears to be the same as the difference $\tan \beta_{r}(\theta)-\tan \beta_{r}(0) \simeq 0.08$ for the angle of repose $\beta_{r}$. Thus, the deviation $\tan \beta-\tan \beta_{r}(\theta)$ or $\tan \beta-\mu_{s}(\theta)$ appears to be the control parameter of the dynamics of water-immersed granular avalanches, which characterizes the out-of-equilibrium state of the system. This will be explained in the modelling presented in next section.

\section{Modelling the avalanche}

Let us now present a modelling of our experimental configuration. It is based on the analysis developed for steady dense flows of dry granular matter in GDR MiDi (2004), and adapted recently either for dry confined flows by Jop et al. (2005) or for water-immersed grains by Cassar et al. (2005). The key point is that for a granular material exhibiting a simple shear with shear rate $\gamma$ and the confining granular pressure $\tau_{z z}=P_{g}$, the shear stress $\tau_{x z}$ obeyes a Coulomb relation

$$
\tau_{x z}=\mu(I) P_{g},
$$

where the friction coefficient $\mu$ depends on a single dimensionless parameter $I$ and where the solid volume fraction $\phi$ is assured to be the same over the whole flowing layer. The inertial number $I$ can be viewed as the ratio of two time scales: the typical flow time scale $1 / \gamma$, and the typical time scale for one grain to fall from on top of another grain to the hole below it. The typical falling time scale is the free fall time $t_{f f} \sim d\left(\rho_{g} / P_{g}\right)^{1 / 2}$ for dry grains of diameter $d$ and density $\rho_{g}$, but corresponds to the viscous falling time $t_{v f} \sim \eta /\left(\alpha P_{g}\right)$ for grains immersed in a viscous liquid of viscosity $\eta$ (Cassar et al. 2005). The coefficient $\alpha$ relates the permeability $k$ of the corresponding granular medium to the grain diameter $d: \alpha=k / d^{2}$. Whatever the 
regimes, the dependence of the friction coefficient $\mu$ on the parameter $I$ is fitted well by the relation (Cassar et al. (2005))

$$
\mu(I)=\mu_{s}+\frac{\mu_{m}-\mu_{s}}{1+I_{0} / I} .
$$

The coefficient $\mu$ varies continuously from the lower asymptotic value $\mu_{s}$ for slow flows with low shear rate $\gamma$ (i.e. for $I \ll 1$ ) towards the maximum asymptotic value $\mu_{m}$ for rapid flows with high shear rate $\gamma$ (i.e. for $I \gg 1$ ). Note that this kind of analysis does not catch the complex static/flowing transition of granular matter, such as for instance the hysteretic behaviour of granular piles between the two characteristic avalanche angles $\beta_{c}$ and $\beta_{r}$.

\subsection{Confined submarine avalanches}

\subsubsection{Model equations}

Let us apply this theoretical framework to our experimental configuration in a quasi-steady regime. The equilibrium of a slice of immersed grains of solid packing fraction $\phi$ between the upper surface and the depth $z$ confined between two parallel solid walls separated by the gap width $b$ can be written as

$$
0=\tan \beta-\mu_{w} \frac{z}{b}-\mu(I) \text {. }
$$

The first term of the right-hand side corresponds to the driving gravity force whereas the two other terms correspond to resisting friction forces: the solid friction at the lateral walls with the friction coefficient $\mu_{w}$, and the friction at the bottom of the slice due to the shear in the granular pile. Note that this last term depends on the vertical position $z$ as the shear rate $\gamma$ (and thus $I$ ) varies in the depth $z$ of the granular pile. As stated above, the parameter $I=\eta \gamma /\left(\alpha P_{g}\right)$ for liquid immersed grains flowing under gravity can be written

$$
I(z)=\frac{\eta \gamma(z)}{\alpha \phi \Delta \rho g z \cos \beta},
$$

as $P_{g}=\phi \Delta \rho g z \cos \beta$ is the normal stress due to the buoyant weight of the grains.

\subsubsection{Flowing layer thickness}

The thickness $h$ of the flowing layer can be determined by considering the depth where $\gamma(h)=0$, so that $I(h)=0$. In such a case, the last term of equation (4.3) tends towards $\mu_{s}$, and (4.3) leads to

$$
\frac{h(\beta)}{b}=\frac{\tan \beta-\mu_{s}}{\mu_{w}} .
$$

Note that condition (4.5) does not depend on the precise expression chosen for the dynamical friction coefficient $\mu(I)$ but just on the non-zero friction coefficient $\mu_{s}$ at low shear. Equation (4.5) predicts a linear dependence of the thickness of the flowing layer on $\tan \beta$ as observed experimentally in figure 6 . The best linear fit of our data gives $\mu_{w} \simeq 0.37 \simeq \tan 20.3^{\circ}$ and $\mu_{s} \simeq 0.43 \simeq \tan 23.3^{\circ}$. Although both these values depend on the precise criterion chosen to measure experimentally the flowing layer thickness, similar values can be found in the literature (Jop et al. 2005; Taberlet et al. 2003; Cassar et al. 2005). Note that this modelling does not describe the static/flowing transition, so that (4.5) does not predict the angle of repose $\beta_{r}$ nor the corresponding avalanche thickness $h_{r}$ that are found experimentally to be $\beta_{r} \simeq 29^{\circ}\left(\tan \beta_{r} \simeq 0.55\right)$ and $h_{r} \simeq 0.7 \mathrm{~mm}$. 


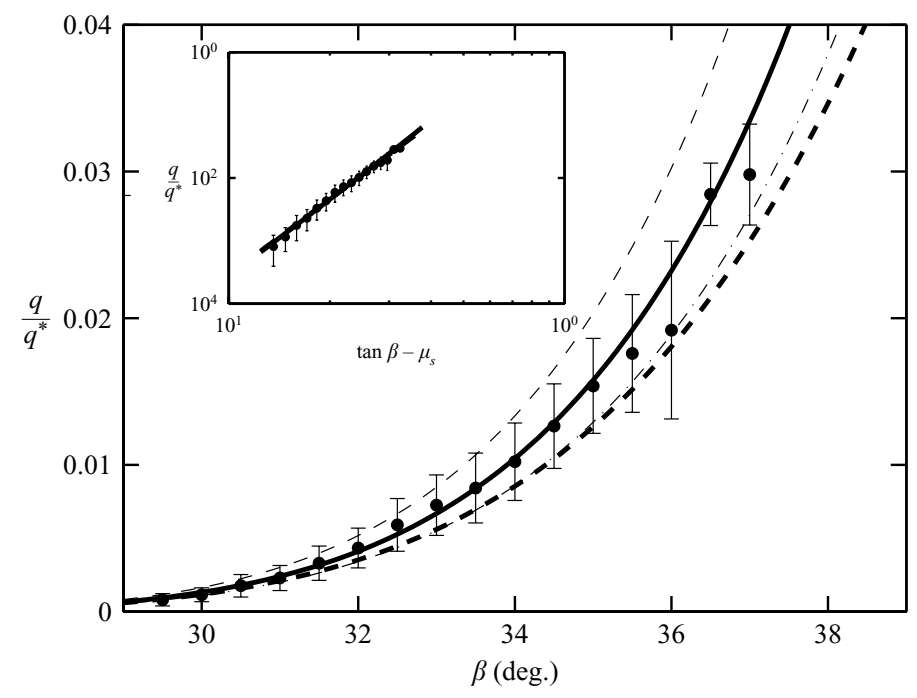

FIgURE 9. Normalized granular flux $q / q^{*}$ as a function of the pile angle $\beta$. Data points (-) correspond to the mean values of the experiments of figure 2 and error bars are the corresponding standard deviations. Lines come from the modelling using equations (4.6) with $\mu_{w}=0.37, \mu_{s}=0.43$, and $\mu_{m}=1(-), \mu_{m}=0.9(--), \mu_{m}=1.1(-\cdot)$, and using the approximate power law (A 3) $q / q^{*} \sim\left(\tan \beta-\mu_{s}\right)^{4}(---)$, with $\mu_{m}=1$. Same data are shown in the inset, using a log-log representation.

\subsubsection{Granular flux}

Equation (4.3) together with (4.2) and (4.4) also allows us to deduce the profile across the depth of the shear rate $\gamma(z)$, thus the velocity profile $v(z)$ and the grain flux per unit width $q$ by successive integration as

$$
\left.\begin{array}{rl}
\gamma(z) & =\gamma^{*} \cos \beta \frac{z}{b}\left(\frac{\mu_{m}-\mu_{s}}{\tan \beta-(z / b) \mu_{w}-\mu_{s}}-1\right)^{-1}, \\
v(z) & =\int_{h}^{z} \gamma(z) \mathrm{d} z, \\
q & =\int_{h}^{0} v(z) \mathrm{d} z,
\end{array}\right\}
$$

where $\gamma^{*}=\alpha \phi I_{0} \Delta \rho g b / \eta$. In the following, we will use the velocity scale $v^{*}=\gamma^{*} b$ and the granular flux scale $q^{*}=\gamma^{*} b^{2}$.

From the set of equations (4.6) we can calculate the granular flux $q$ for a given angle $\beta$ provided $\beta$ is smaller than the upper limit value $\arctan \mu_{m}$. In figure 9 , the normalized granular flux $q / q^{*}$ is plotted by using for $q^{*}$ the same parameter values of $\phi=0.55, \alpha=0.01$ and $I_{0}=1$ as used by Cassar (2005). The other friction parameters have been determined by the fit of our $h$ data $\left(\mu_{w}=0.37\right.$ and $\mu_{s}=0.43$, see $\left.\S 4.1 .2\right)$, except $\mu_{m}$, which will be determined by the fit of our $q$ data. The experimental data for the granular flux $q_{\beta}$ are obtained by averaging the time derivative of the slope relaxations of figure 2. The numerical calculation of $q$ for $\mu_{m}=0.9,1$ and 1.1 is plotted on figure 9 . The curve for $\mu_{m}=1$ fits the experimental data well. This value is a little higher than the range $(0.82,0.87)$ of $\mu_{m}$ found in Cassar $(2005)$. The granular flux increases nonlinearly with $\beta$ since both the surface velocity and the flowing layer thickness $h$ increase with $\beta$. As $\cos \beta$ does not vary as much in the explored 


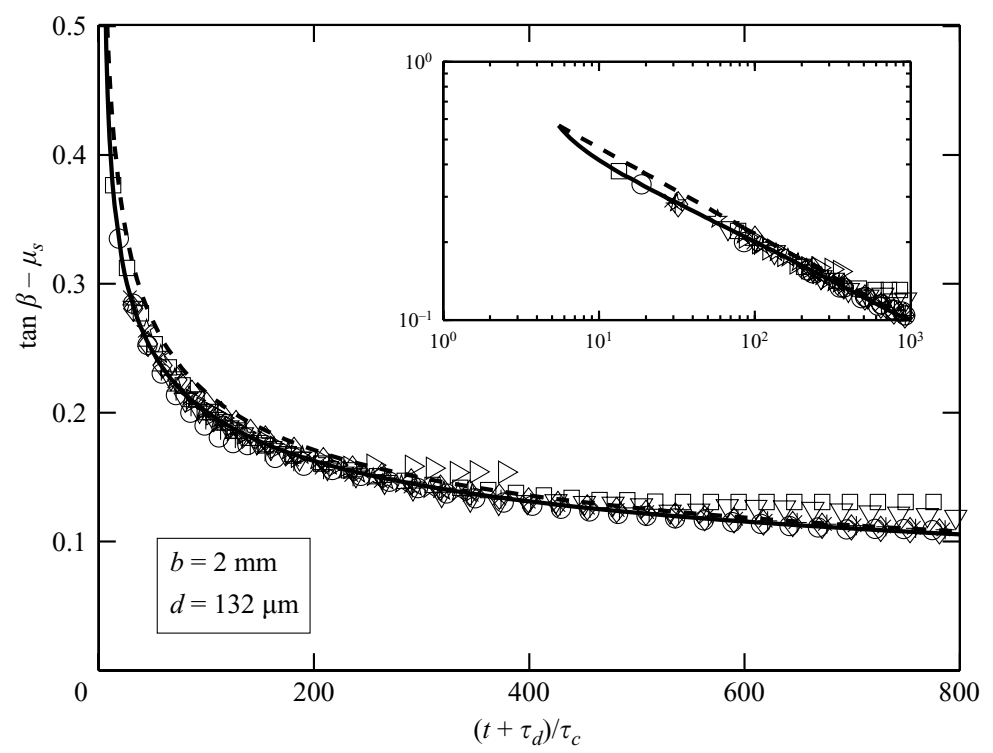

FiguRE 10. Normalized time variation of the pile angle $\beta$. Experimental data (same symbols as in figure 2) are rescaled using best-fitting values of $\mu_{s}=0.434 \pm 0.013$ and $\tau_{c}=29.9 \pm 0.07 \mathrm{~s}$. Numerical modelling (-) using equations (4.6) with the same parameter values as in figure 9 , and analytical approximation (- - ) using equation (A5): $\tan \beta(t)=\mu_{s}+\left[\tau_{c} /\left[t+\tau_{d}\right]\right]^{1 / 3}$. The inset is the log-log plot of the same data.

range of angles, this nonlinear variation corresponds roughly to $q / q^{*} \sim\left(\tan \beta-\mu_{s}\right)^{4}$ as demonstrated in the Appendix (A 3) for small enough flowing layer thicknesses and thus small enough pile angles. Such an analytical scaling (thick dashed line in figure 9) is close to the complete numerical calculation at small angles but deviates significantly at larger angles, by about $25 \%$ at $\beta \simeq 37^{\circ}$. For even higher angles, the analytical scaling underpredicts the $q$ values even more as the complete calculation diverges when $\beta$ approaches $\arctan \mu_{m} \simeq 45^{\circ}$ from below. Note that Jop et al. (2005) found a similar scaling of the granular flux $q \sim\left(\tan \beta-\mu_{s}\right)^{7 / 2}$ for dry confined steady flows, with a slightly different exponent. This slight difference arises from the differences in the velocity profiles between the dry case and the liquid-immersed case, as the inertial parameter $I$ does not have the same expression in the two cases. Note also that when integrating (4.6) $q$ is assumed to tends toward 0 when $\beta$ tends toward $\arctan \mu_{s} \simeq 23.3^{\circ}$ but the flow experimentally stops at an angle of repose $\beta_{r}>\arctan \mu_{s}$, for which the modelling predicts a weak but non-zero value of $q$.

\subsubsection{Slope relaxation}

Since the time derivative of the pile angle $\beta$ is related to the granular flux $q$ by equation (2.1), we can calculate by numerical integration any $\beta(t)$ curve starting from a given initial value $\arctan \mu_{s}<\beta_{0}<\arctan \mu_{m}$. Each experimental curve $\beta(t)$ of figure 2 can thus be fitted by the model with the same parameter values but slightly different $\mu_{s}$ values: $\mu_{s}=0.434 \pm 0.013$, which correspond closely to the $\mu_{s}$ value 0.43 found previously with the flowing layer thickness measurements. All the $\beta(t)$ data can be seen in the normalized plot of figure 10 together with the full master numerical curve (solid line). For this, each experimental curve has to be shifted by a time lag $\tau_{d}$ depending on the corresponding initial angle $\beta_{0}\left(\tau_{d}\right.$ increases for decreasing $\left.\beta_{0}\right)$. One can see in figure 10 that the data are very close to the model except that 
experimentally the avalanches stop at an angle of repose larger than $\arctan \mu_{s}$ : the experimental curves then deviate from the model curve with a plateau after the avalanche stop. The dispersion in the plateau values corresponds to the experimental fluctuation of the angle of repose. The slope relaxations $\beta(t)$ do not correspond to an exponential decrease but roughly to a power law. Indeed, as detailed in the Appendix, analytical calculations are possible under some restrictions. The key point is that the slope relaxation corresponds to the scaling $\left(\left(t+\tau_{d}\left(\beta_{0}\right)\right) / \tau_{c}\right)^{-1 / 3}$ (A 5), where $\tau_{c}$ is a characteristic time proportional to $L^{2} / q^{*}(\mathrm{~A} 6)$. This scaling (dashed line in figure 10) is very close to the complete calculation (solid line). The $\tau_{c}$ values can be inferred from the previous fitting $\mu_{s}$ values: $\tau_{c}=29.9 \pm 0.07 \mathrm{~s}$.

\subsubsection{Avalanche time duration}

As the modelling predicts an avalanche slope relaxation toward the low limit value $\arctan \mu_{s}$ with a vanishing granular flux, the asymptotic value $\arctan \mu_{s}$ would be reached for an infinite time. However, since the slope relaxation stops experimentally at the angle of repose $\beta_{r}>\arctan \mu_{s}$, the experimental avalanche time duration should be finite, with the scaling (A 8):

$$
T=\tau_{c}\left[\frac{1}{\left(\tan \beta_{r}-\mu_{s}\right)^{3}}-\frac{1}{\left(\tan \beta_{0}-\mu_{s}\right)^{3}}\right] .
$$

Expression (4.7) is much more complex than the one proposed by Courrech du Pont et al. (2003a) as their scaling $T \sim L / V_{S t}$ was independent of the avalanche amplitude. However, Courrech du Pont et al. (2003a) report results on natural avalanches of small amplitude from $\beta_{c}$ to $\beta_{r}$ unlike the present case where the system is driven far from equilibrium. One interesting point in the present study is that the avalanche duration increases with the starting angle $\beta_{0}$. At large initial angle $\beta_{0}, T$ is assumed to tend towards the asymptotic value $T=\tau_{c}\left(\tan \beta_{r}-\mu_{s}\right)^{-3}=(5 \pm 1)$ hours which corresponds closely to the measured experimental time duration $T=(5.5 \pm 1)$ hours.

\subsubsection{Influence of the gap thickness and grain diameter}

In the modelling, we can see that the granular flux $q$ per unit width is proportional to $b^{3}$ and independent of the grain size (A 3). The flux increase with $b$ is intuitive as one can easily imagine that wall friction is less important for wider gap cell. The $b$ dependence ( $b^{4}$ for the total flux) that comes from the strong localization of the flowing layer on the scale $b$ resembles that of the Hagen-Poiseuille law for the flow of a classical viscous fluid in a duct of transverse size $b$. As in the Hagen-Poiseuille law where there is an equilibrium between the driving pressure gradient and the resisting viscous forces of the fluid, there is here an equilibrium between the driving pressure forces of gravity and the resisting viscous and granular friction forces. The non- $d$-dependence of $q((\mathrm{~A} 2)$ and (A3)) is not intuitive as one could imagine that larger grains would flow faster as for a single falling grain or a classical sedimenting suspension. This surprising result comes from the fact that the time scale used in the present model is $t_{v f} \sim \eta /\left(\alpha P_{g}\right)$, which is independent of the grain diameter as the granular pressure $P_{g}$ is proportional to the depth and thus to the cell gap $b$. We have thus done other experiments by varying the cell gap $b$ and the grain diameter $d$ to test the predictions of the model. The results are plotted in figure 11 for avalanche slope relaxations in a larger cell of gap thickness $b=5 \mathrm{~mm}$. The model again fits the experimental data quite well with $\mu_{s}=0.385 \pm 0.07$ giving $\tau_{c}=1.91 \pm 0.02 \mathrm{~s}$. This value is 15.7 times smaller than the $\tau_{c}$ value found for $b=2 \mathrm{~mm}$ and very close to the $(5 / 2)^{3} \simeq 15.6$ predicted factor. Note that for this larger gap, the experimental angle of repose $\beta_{r}=25.7^{\circ} \pm 0.4^{\circ}$ is smaller (Courrech du Pont et al. 2003b) due to smaller wall 


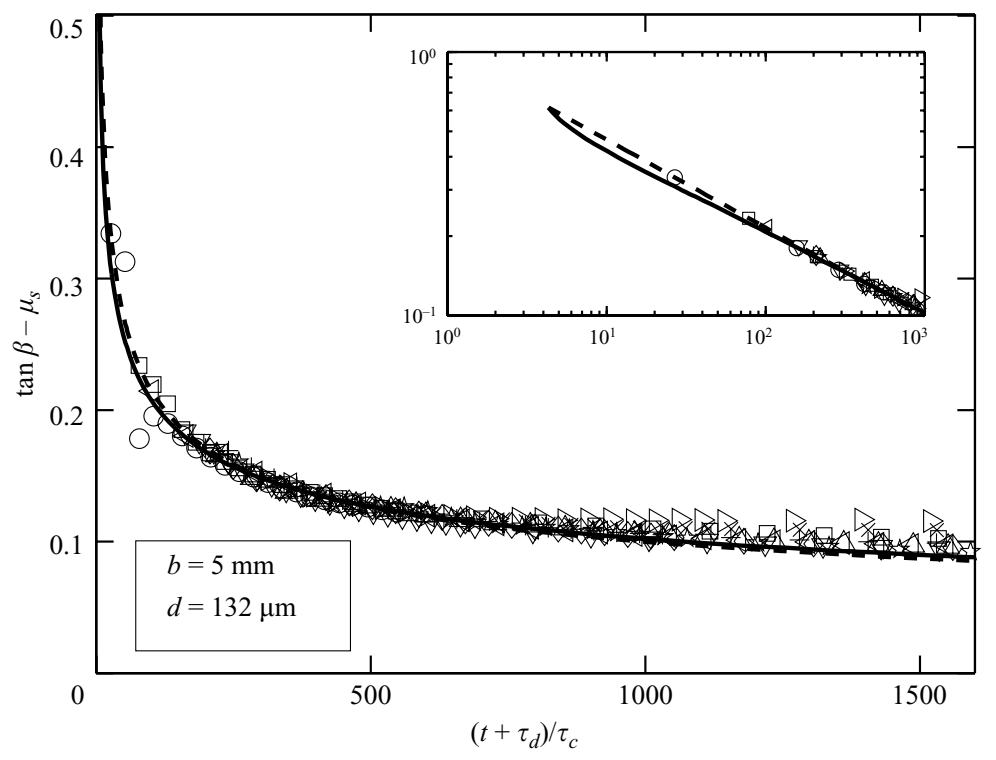

FiguRE 11. Normalized time variation of the pile angle $\beta$, with the same glass beads as in figure $10(d=132 \mu \mathrm{m})$, but with a wider gap $b=5 \mathrm{~mm}$. Experimental data are rescaled using best-fitting values of $\mu_{s}=0.385 \pm 0.007$ and $\tau_{c}=(1.91 \pm 0.02) \mathrm{s}$ for the different curves starting from $\beta_{0}=27.7^{\circ}(\times), \beta_{0}=27.8^{\circ}(\triangleright), \beta_{0}=28.9^{\circ}(+), \beta_{0}=29.1^{\circ}(\triangle), \beta_{0}=29.2^{\circ}(\diamond), \beta_{0}=29.5^{\circ}$ (次), $\beta_{0}=29.8^{\circ}(\nabla), \beta_{0}=30.9^{\circ}(\triangleleft), \beta_{0}=31.5^{\circ}(\square), \beta_{0}=35.5^{\circ}$ (०). Numerical modelling (一) using equations (4.6) and analytical approximation (- - ) using equation (A5). The inset is the log-log plot of the same data.

effects, and the experimental curves $\beta(t)$ thus cover angle values closer to $\arctan \mu_{s}$ in a longer normalized time $\left(t+\tau_{d}\right) / \tau_{c}$. The measured time duration of avalanches here is $T=(80 \pm 30) \mathrm{min}$, a little larger than the predicted values $T=(40 \pm 20) \mathrm{min}$.

We have also done other experiments now varying the grain diameter $d$. The results for the avalanche slope relaxations are plotted in figure 12 for the thickness $b=5 \mathrm{~mm}$ but with larger grains of diameter $d=(360 \pm 40) \mu \mathrm{m}$. Again, the agreement with the model is quite good with the fitting parameters $\mu_{s}=0.444 \pm 0.005$ giving $\tau_{c}=(1.88 \pm 0.02) s$. This value of $\tau_{c}$ is very close to the value $\tau_{c}=(1.91 \pm 0.02) s$ found previously for the smaller grains $(d=132 \mu \mathrm{m})$ in the same gap cell, meaning that the avalanche process is indeed independent of the grain size as predicted by the model. The measured time duration of avalanches is much shorter here, $T=(6 \pm 1)$ min, which is close to the predicted value $T=(7 \pm 3) \mathrm{min}$. Note that even if the characteristic time values $\tau_{c}$ are the same for the two different grain diameters in the same cell gap, the avalanche time duration is smaller for the larger grains as the angle of repose is larger $\left(\beta_{r}=31.5^{\circ} \pm 1^{\circ}\right)$ due to larger wall effects.

\subsubsection{Velocity profiles}

The velocity profiles across the depth can also be calculated by the set of equations (4.6). An example of such a profile for the pile angle $\beta=34.3^{\circ}$ together with the experimental data are reported in figure 13. Though the qualitative trend is the same, i.e. a maximal velocity at the free surface $(z=0)$ and a decreasing velocity further in, there is a quantitative mismatch: the model predicts that the velocity is strictly zero at the precise depth $z=h(h / b \simeq 0.67$ in figure 13) whereas the experimental data decrease smoothly towards zero with an exponential tail. Again, this mismatch shows that the modelling fails in reproducing the complex and hysteretic 'liquid-solid' 


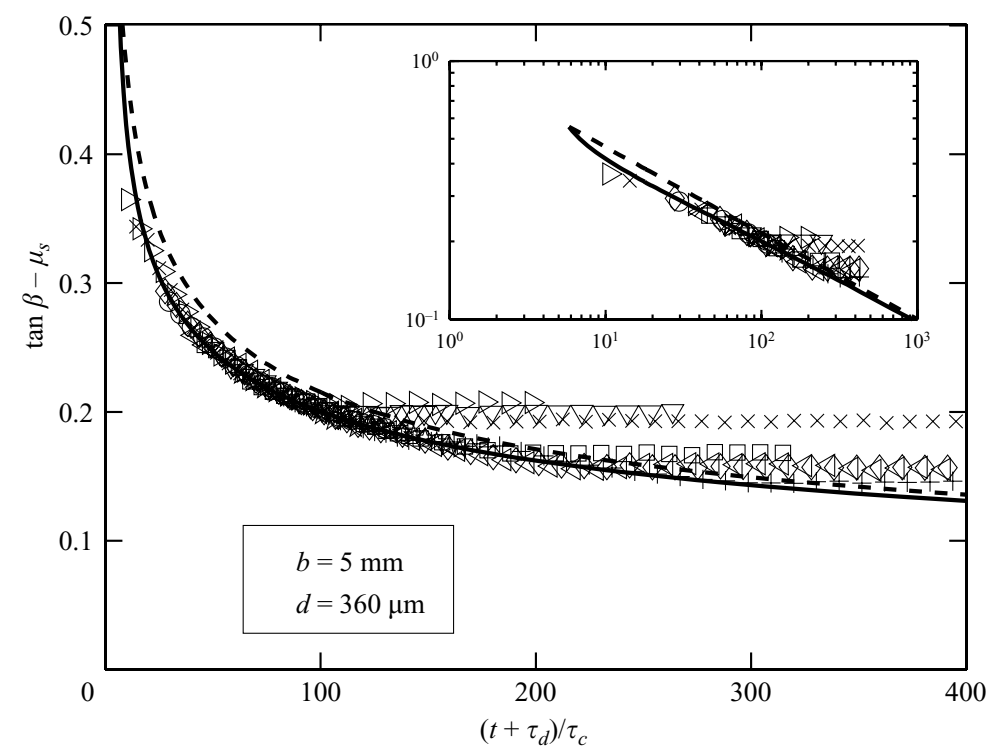

FIGURE 12. Normalized time variation of the pile angle $\beta$, for the same gap $b=5 \mathrm{~mm}$ as in figure 11 but with a larger grain diameter $d \simeq 360 \mu \mathrm{m}$. Experimental data are rescaled using best-fitting values of $\mu_{s}\left(\mu_{s}=0.444 \pm 0.005\right)$ and $\tau_{c}=(1.88 \pm 0.02) \mathrm{s}$ for the different curves starting from $\beta_{0}=33.1^{\circ}(+), \beta_{0}=34.0^{\circ}(\nabla), \beta_{0}=34.2^{\circ}(\triangleleft), \beta_{0}=34.7^{\circ}(\square), \beta_{0}=35.4^{\circ}(\triangleleft)$, $\beta_{0}=36.0^{\circ}(\circ), \beta_{0}=36.3^{\circ}(\diamond), \beta_{0}=38.4^{\circ}(\times), \beta_{0}=39.2^{\circ}(\triangleright)$. Numerical modelling $(-)$ using equations (4.6) and analytical approximation (- - ) using equation (A5). The inset is the log-log plot of the same data.

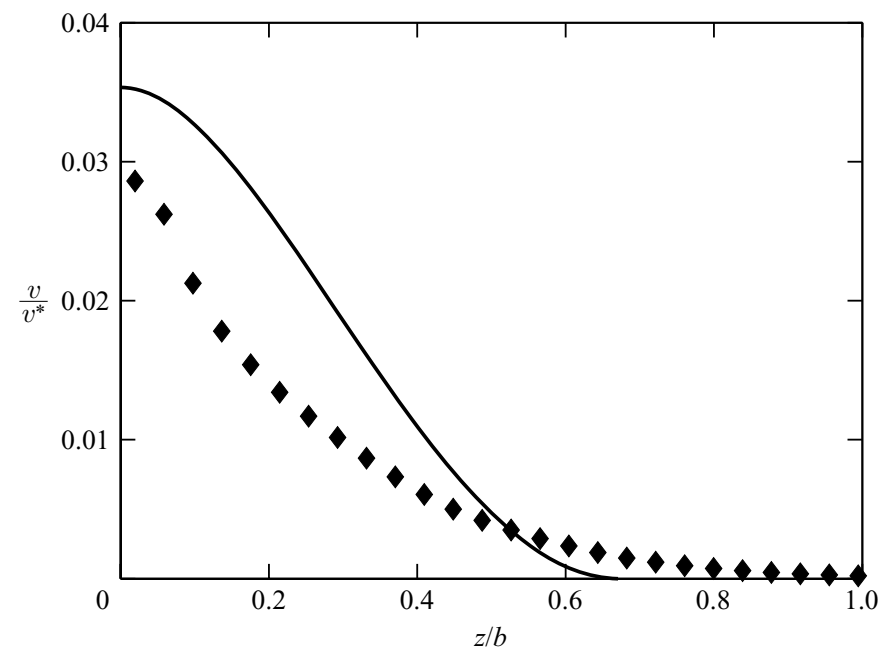

FIGURE 13. Normalized granular velocity profile for the pile angle $\beta=34.3^{\circ}$. Experimental data $(\diamond)$ and modelling $(-)$ using equations (4.6).

transition. The experimental tail, also observed for dry granular avalanches on piles and often referred as 'creeping flow', is not well understood for the moment. Another mismatch between theoretical and experimental velocity profiles is observed at the vicinity of the free surface: the maximum normalized velocity is similar $(0.035$ instead of 0.03 ), but the modelling predicts $\gamma=0$ at the free surface $z=0$ whereas this is not 


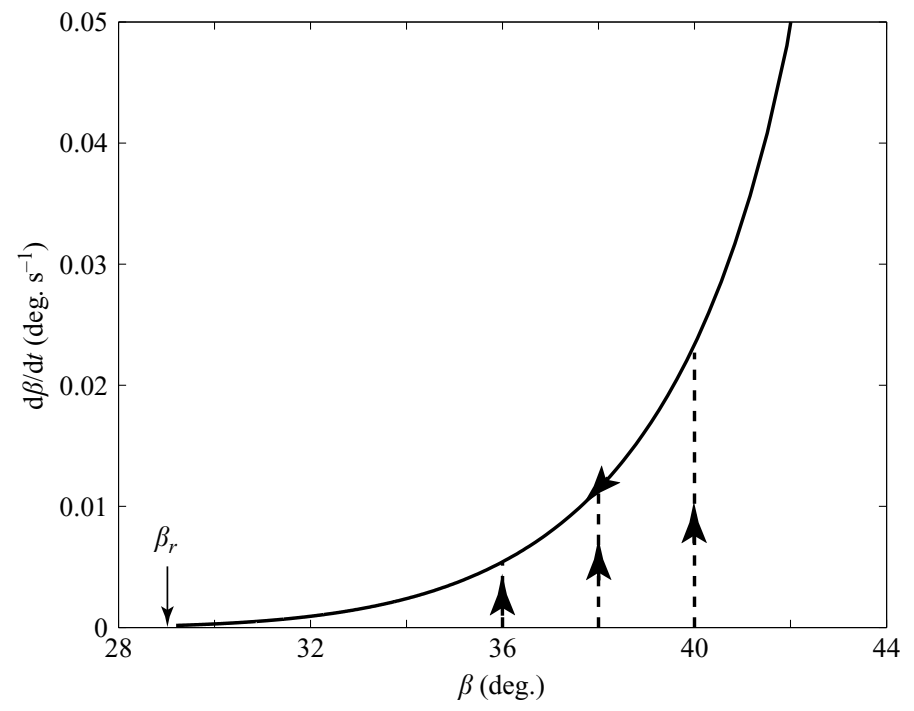

Figure 14. Evolution of $\mathrm{d} \beta / \mathrm{d} t$ versus $\beta$ for avalanches of different initial angles $\beta_{0}$. Dotted lines are for the transient parts.

the case for the experimental data (figure $8 a$ ). However, the experimental data show a clear inflection point and a decrease of the shear rate close to the free surface. Note that the experimental velocity profiles are measured at the wall whereas the velocity profiles calculated in the modelling arise from an averaging over the entire width. This may explain why the measurements are lower than the model, and can also introduce some distortion as we have checked experimentally that the global granular flux $q_{\beta}$ measured by the angle variation is different from the granular flux $q_{p i v}$ measured by PIV means at the wall: the ratio $q_{\beta} / q_{\text {piv }}$ increases roughly from 1 for $\beta \sim \beta_{r}$ to 5 for $\beta \sim \arctan \mu_{m}$. This means that the velocity profile $v(z)$ varies across the width of the channel, with a slip velocity at the wall that depends on the pile angle. This behaviour is clearly not described well by our width-averaged modelling.

\subsubsection{Initial transient regime}

Let us return to the initial transient regime. Starting from the initial angle $\beta_{0}$ with a zero granular flux, the granular flux increases quickly toward its saturated value $q(\beta)$ that we have already determined. This transient phase can be modelled by adding an acceleration term on the left-hand side of equation (4.3). The calculation shows a transient time for the granular flux increase of the order of $0.1 \mathrm{~s}$ instead of the few seconds observed experimentally (see figure 3). Although the model fails to reproduce correctly the very first instants of the avalanche dynamics, we can however plot the avalanche path in the $(\beta, \mathrm{d} \beta / \mathrm{d} t)$-plane, since $\mathrm{d} \beta / \mathrm{d} t$ is directly related to the granular flux $q$. Starting from a given angle $\beta_{0}$, the granular flux and thus $\mathrm{d} \beta / \mathrm{d} t$ increase quickly with almost no variation of $\beta$ along the vertical dotted line sketched in figure 14, until they reach their saturated value at the solid line. Then the pile slope $\beta$ slowly decreases in a quasi-steady way following the solid line.

\subsection{Influence of a water counter-flow}

When a water counter-flow characterized by the Shields number $\theta$ is applied along the granular pile, the fluid forces have to be added to the force balance. In our Hele-Shaw 

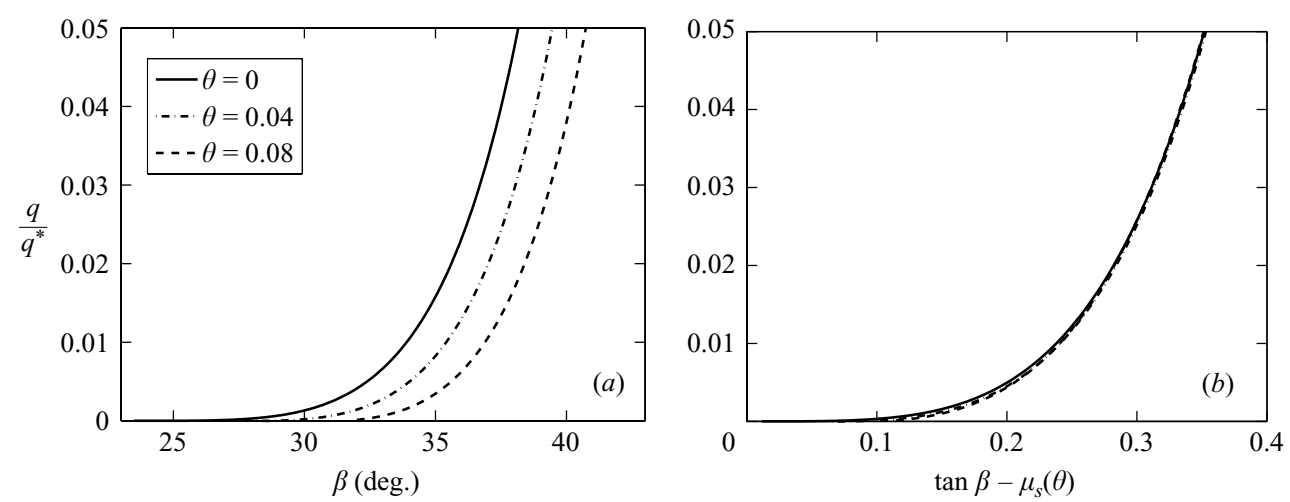

Figure 15. (a) Normalized granular flux $q / q^{*}$ as a function of the pile angle $\beta$ for different water counter-flows of Shields number $\theta=0(-), \theta=0.04$ (- * -) and $\theta=0.08$ (- - -). (b) The same data plotted as a function of $\tan \beta-\mu_{s}(\theta)$ with $\mu_{s}(0)=0.434, \mu_{s}(0.04)=0.48$ and $\mu_{s}(0.08)=0.51$.

configuration, these fluid forces have been shown to have two origins (Loiseleux et al. 2005):

(i) the shear stress $\tau_{x z}=\Delta \rho g d \theta$ related to the water shear at the water/grains interface,

(ii) the longitudinal pressure gradient $\partial \tau_{x x} / \partial x \simeq 3.7(\Delta \rho g d \theta / b)$ existing in the porous phase and which is also constant along the cell (independent of the $x$ position).

The equilibrium of a slice of material of thickness $z$ can then be written

$$
0 \simeq \tan \beta-\mu_{w} \frac{z}{b}-\mu(I)-\theta d \frac{1+3.7 z / b}{\phi z \cos \beta},
$$

where the last extra term in comparison to equation (4.3) represents the fluid resisting forces having the two origins mentioned above. For a given slope angle $\beta$ and a given Shields number $\theta$, the thickness $h$ of the flowing layer is determined by the quadratic relation

$$
0 \simeq \tan \beta-\mu_{w} \frac{h}{b}-\mu_{s}-\theta d \frac{1+3.7 h / b}{\phi h \cos \beta} .
$$

For large angle values $\beta$, the flowing layer $h$ is thick enough $(h \sim b)$ so that the fluid force term is reduced to its longitudinal pressure gradient origin. The flowing layer thickness $h$ can thus be approximated by

$$
\frac{h}{b} \simeq \frac{\tan \beta-\left(\mu_{s}+3.7(\theta d / \phi b \cos \beta)\right)}{\mu_{w}} .
$$

As $\cos \beta$ does not vary much around the value $\cos 35^{\circ} \simeq 0.8$, the predicted flowing layer thickness $h$ depends quasi-linearly on $\tan \beta$, which satisfactorily reproduces the experimentally found linear variation (figure $8 b$ ). It is as if the limit friction value $\mu_{s}$ had to be increased by a value proportional to the Shields number $\theta$ : $\mu_{s}(\theta) \simeq \mu_{s}+4.6 \theta d / \phi b$. In the same way that (4.3) leads to the set of equations (4.6) for $\theta=0$, (4.8) leads to a similar set of equations that allows, for $\theta \neq 0$, calculations of the shear rate $\gamma(z)$, the velocity profile $v(z)$, and the granular flux $q$ per unit width. The numerical results for $q$ are shown in figure 15(a) for two different values of the Shields number $\theta=0.04$ and $\theta=0.08$, together with the no-flow case $\theta=0$. Numerical data show that $q$ decreases with increasing $\theta$ for a given slope angle $\beta$, meaning that 
the water counter-flow slows down the avalanche. This is clearly due to the added resisting effect of the fluid. Like the flowing layer thickness, the granular flux $q$ is also governed by $\tan \beta-\mu_{s}(\theta)$ as the different curves collapse in figure $15(b)$. Thus the parameter $\tan \beta-\mu_{s}(\theta)$ appears to be the control parameter of the system that characterizes its out-of-equilibrium state. As we already found experimentally that the deviation $\mu_{s}(\theta)-\mu_{s}(0)$ between the values of the friction coefficient is roughly the same as the deviation $\tan \beta_{r}(\theta)-\tan \beta_{r}(0)$ between the values of the angle of repose (figure $8 b$ ), this explains why the experimental velocity profiles coincide (figure $8 a$ ) for same values of $\tan \beta-\tan \beta_{r}(\theta)$, which can be viewed also as a good experimental control parameter.

\section{Conclusion}

In this paper, we have presented experimental results concerning water-immersed avalanches of small grains (in the so-called viscous regime) in a long and narrow channel of gap $b$, with or without a water counter-flow. Whatever the initial pile angle, even much larger than the maximum angle of stability, the slope relaxation corresponds to a quasi-steady motion after a short transient. The pile slope $\beta$ has been shown to be the sole control parameter for the granular flow. When a water counterflow characterized by the Shields number $\theta$ is imposed, the dynamics of the granular flow is slowed down, but all the results are similar if one considers the deviation of the pile angle from the corresponding angle of repose $\beta_{r}(\theta)$. These results have been modelled well using a unique granular friction coefficient depending on the local shear rate, together with taking into account the solid friction due to the lateral walls and also the fluid friction due to the possible water flow. Calculations have led to a granular flux scaling $q \sim\left(\tan \beta-\mu_{s}\right)^{4}$, which agrees satisfactorily with the experimental data. A consequence of this power-law scaling of $q$ is that the pile slope evolves in time as $t^{-1 / 3}$. Another key point is that the granular flux increases nonlinearly with the gap thickness of the cell $\left(q \sim b^{3}\right)$ and seems to be independent of the grain diameter. The characteristic time of large-amplitude avalanches on waterimmersed granular piles of small grains driven far from equilibrium thus decreases nonlinearly with the cell gap $\left(\sim b^{-3}\right)$ and is independent of the grain diameter.

The authors are grateful to Olivier Pouliquen and Daniel Lhuillier for fruitful discussions. Fréderic Moisy, Raphaël Fischer and Sylvain Courrech du Pont are also acknowledged for their assistance concerning the use of PIV technique. This work has been supported by the ACI Jeunes Chercheurs 2114 of the Ministère Français de la Recherche.

\section{Appendix}

For convenience, let us introduce the parameter $h_{m}=b\left(\mu_{m}-\mu_{s}\right) / \mu_{w}$ corresponding to the maximal flowing layer thickness obtained for the largest slope angle $\beta=\arctan \mu_{m}$, beyond which the rheology is no longer valid. Integrating equation (4.6) twice with the boundary conditions $\gamma(h)=0$ and $v(h)=0$, gives the following analytical expression for the granular flux $q$ per unit width:

$$
q(h)=q^{*} \cos \beta \frac{h_{m}^{3}}{b^{3}}\left[-\frac{h}{h_{m}}\left(\frac{1}{3}\left(\frac{h}{h_{m}}\right)^{2}-\frac{3}{2} \frac{h}{h_{m}}+1\right)-\left(1-\frac{h}{h_{m}}\right)^{2} \ln \left(1-\frac{h}{h_{m}}\right)\right],
$$

where $q^{*}=\alpha \phi I_{0} \Delta \rho g b^{3} / \eta$. 
For small enough flowing layer thickness $h$ (when compared to the maximal thickness $h_{m}$ ) corresponding to a moderate value of the pile angle, (A 1) can be written at first order as

$$
q(h)=q^{*} \frac{\cos \beta}{12 b^{3} h_{m}} h^{4}+O\left(h^{5}\right)
$$

The granular flux $q$ appears to be a power function of $h$. As the flowing layer thickness $h$ is proportional to $\tan \beta-\mu_{s}$ as shown by (4.5), the granular flux may be written as

$$
q(\beta) \simeq \frac{q^{*} \cos \beta}{12 \mu_{w}^{3}\left(\mu_{m}-\mu_{s}\right)}\left(\tan \beta-\mu_{s}\right)^{4} .
$$

Since the time derivative of the pile angle $\beta$ is related to the granular flux $q$ by equation (2.1) and considering a quasi-steady process, we can calculate the elementary angle variation $\mathrm{d} \beta$ in the elementary time $\mathrm{d} t$ by

$$
-\frac{\mathrm{d} \beta}{\cos \beta\left(\tan \beta-\mu_{s}\right)^{4}} \simeq \frac{2 q^{*}}{3 \mu_{w}^{3}\left(\mu_{2}-\mu_{s}\right) L^{2}} \mathrm{~d} t .
$$

As $\cos \beta$ does not vary much in the explored range of plie angles, (A 4) can be integrated. Given an initial slope $\beta_{0}$, the time evolution of $\beta$ may thus be approximated by

$$
\tan \beta(t) \simeq \mu_{s}+\left(\frac{\tau_{c}}{t+\tau_{d}\left(\beta_{0}\right)}\right)^{1 / 3}
$$

with the two time constants

$$
\begin{gathered}
\tau_{c}=\frac{\mu_{w}^{3}\left(\mu_{m}-\mu_{s}\right) L^{2} \overline{\cos \beta}}{2 q^{*}}, \\
\tau_{d}\left(\beta_{0}\right)=\frac{\tau_{c}}{\left(\tan \beta_{0}-\mu_{s}\right)^{3}} .
\end{gathered}
$$

The parameter $\tau_{d}\left(\beta_{0}\right)$ is the time lag by which each $\beta(t)$ curve for a given initial slope $\beta_{0}$ has to be shifted in order to fit the main curve (which starts at $\arctan \mu_{m}$ ), whereas $\tau_{c}$ is an intrinsic time scale of the granular flow. This modelling predicts a decrease of $\beta$ toward the asymptotic value $\arctan \mu_{s}$ in an infinite time. If one defines the time duration $T$ of the avalanche as the time needed for the pile to relax from $\beta_{0}$ to the angle of repose $\beta_{r}$, the expression for $T$ is

$$
T \simeq \frac{\tau_{c}}{\left(\tan \beta_{r}-\mu_{s}\right)^{3}}-\tau_{d}\left(\beta_{0}\right) .
$$

\section{REFERENCES}

AlLEN, J. R. L. 1970 The avalanching of granular solids on dune and similar slopes. J. Geol. 78, 326-351.

Andreotti, B. \& Doundy, S. 2001 Selection of velocity profile and flow depth in granular flows. Phys. Rev. E 63, 031305.

Armanini, A., Capart, H., Fraccarollo, L. \& Larcherm, M. 2005 Rheological stratification in experimental free-surface flows of granular liquid mixtures. J. Fluid Mech. 532, 269-319.

Beavers, G. S. \& JosePh, D. D. 1967 Boundary conditions at a naturally permeable wall. J. Fluid Mech. 30, 197-207.

Bonamy, D., Daviaud, F. \& Laurent, L. 2002 Experimental study of granular flows via a fast camera: a continuous description. Phys. Fluids 14, 1666-1673.

CARrigy, M. A. 1970 Experiments on the angles of repose of granular materials. Sedimentology 14, $147-158$. 
CASSAR, C. 2005 Etude expérimentale des écoulements granulaires immergés. PhD thesis, Université de Provence.

Cassar, C., Nicolas, M. \& Pouliquen, O. 2005 Submarine granular flows down inclined planes. Phys. Fluids 17, 103301.

Courrech, S., Fischer, R., Gondret, P., Perrin, B. \& Rabaud, M. 2005 Instantaneous velocity profile during granular avalanches. Phys. Rev Lett. 94, 048003.

Courrech du Pont, S., Gondret, P., Perrin, B. \& Rabaud, M. 2003a Granular avalanches in fluids. Phys. Rev. Lett. 90, 044301.

Courrech du Pont, S., Gondret, P., Perrin, B. \& Rabaud, M. $2003 b$ Wall effects on granular heap stability. Europhys. Lett. 61, 492-498.

da Cruz, F., Emam, S., Prochnow, M., Roux, J.-N. \& Chevoir, F. 2005 Rheophysics of dense granular materials: Discrete simulation of plane shear flows. Phys. Rev. E 72, 021309.

Davis, R. H., Serayssol, J.-M. \& Hinch, E. J. 1986 The elastohydrodynamic collision of two spheres. J. Fluid Mech. 163, 479-497.

Doppler, D. 2005 Stabilité et dynamique de pentes granulaires sous-marines. PhD thesis, Université Paris Sud-11.

GDR MiDi 2004 On dense granular flows. Eur. Phys. J. E 14, 341-365.

Gondret, P., Lance, M. \& Petit, L. 2002 Bouncing motion of spherical particles in fluids. Phys. Fluids 14, 643-652.

Gondret, P., Rakotomalala, N., Rabaud, M., Salin, D. \& Watzky, P. 1997 Viscous parallel flows in finite aspect ratio Hele-Shaw cell: analytical and numerical results. Phys. Fluids 9, 1841-1843.

Hunter, R. E. 1985 Subaqueous sand flow cross-strata. J. Sedimentary Petrol. 55, 886-894.

Jain, N., Ottino, J. \& Lueptow, R. M. 2004 Effect of interstitial fluid on a granular flowing layer. J. Fluid Mech. 508, 23-44.

Jop, P., Forterre, Y. \& Pouliquen, O. 2005 Crucial role of side walls for granular surface flows: consequences for the rheology. J. Fluid Mech. 541, 167-192.

Joseph, G. G., Zenit, R., Hunt, M. L. \& Rosenwinkel, A. M. 2001 Particle-wall collisons in a viscous fluid. J. Fluid Mech. 433, 329-346.

Josserand, C., LAgree, P. \& Lhuillier, D. 2004 Stationary shear flows of dense granular materials: a tentative continuum modelling. Eur. Phys. J. E 14, 127-135.

Loiseleux, T., Gondret, P., Rabaud, M. \& Doppler, D. 2005 Onset of erosion and avalanche for an inclined granular bed sheared by a continuous laminar flow. Phys. Fluids 17, 103304.

Lueptow, R. M., Akonur, A. \& Shinbrot, T. 1999 PIV for granular flows. Exps. Fluids 28, 183-186.

Mills, P., Loggia, D. \& Texier, M. 1999 Model for stationary dense granular flow along an inclined wall. Europhys. Lett. 45, 733-738.

Rajchenbach, J. 2003 Dense, rapid flows of inelastic grains under gravity. Phys. Rev. Lett. 90, 144302.

Taberlet, N., Richard, P., Valance, A., Losert, W., Pasini, J. M., Jenkins, J. T. \& Delannay, R. 2003 Superstable granular heap in a thin channel. Phys. Rev. Lett. 91, 264301. 